\title{
Recent Image Processing Techniques in Forensic Odontology - A Systematic Review
}

\author{
Rameswari Poornima Janardanan*1 and Rajasvaran Logeswaran ${ }^{2}$ \\ ${ }^{1}$ Riyadh Elm University, Riyadh, Kingdom of Saudi Arabia, \\ ${ }^{2}$ Asia Pacific University of Technology and Innovation, 57000 Kuala Lumpur, Malaysia \\ Received: February 22, 2018; Published: March 06, 2018
}

*Corresponding author: Rameswari Poornima Janardanan, Riyadh Elm University,Riyadh,Saudi Arabia, Tel: 533643343; Email: ahlambaha@hotmail.com

\begin{abstract}
Image processing is a strong tool aiding medical and forensic research. This paper covers a systematic review in the area of dental image analysis applied to forensic odontology. The interpretations of medical images rely hugely on human involvement and the human perception of the details present in it, and the interpretation of the fine details in various contrast situations present in a medical image is indeed a challenge as typical radiographs obtained from a regular radiograph acquisition device may only be of average quality. Various standardized scientific tools that have been designed by researchers, scholars and software developers to address this type of shortcomings in a medical radiograph, targeted to minimize the possible human error in predicting the right diagnosis and treatment solely on the basis the human visual perception, are explored.
\end{abstract}

Keywords: Forensic odontology; Medical Image Analysis; Image Segmentation; Systematic Review; Dental age Estimation; Dental Biometrics

Abbreviations: DOAJ: Directory of Open Access Journals; PRISMA: Preferred Reporting Items for Systematic Reviews and Meta-Analyses; AI: Artificial Intelligence; ANNs: Artificial Neural Networks

\section{Introduction}

Forensic odontology involves the management, examination, evaluation and presentation of dental evidence in criminal or civil proceedings, all in the interest of justice [1]. It has the ability to bring justice where dental remains are the only available evidence [2]. There are fields such as computer vision whose ultimate goal is to use computers to emulate human vision, including learning and being able to make inferences and take actions based on visual inputs [3]. The aim of this systematic review is to provide an overview towards state of the art dental image processing methods used in forensic odontology because of their potential importance in the clinical and forensic fields. The relevance of this review is grounded in the need to recommended effective methods of image processing for dental age estimation and human identification with the following characteristics: simple, fast, non-invasive, inexpensive, reproducible and accurate; that can be systematically used in different academic and forensic scenarios.

\section{Methodology}

There have been many trials to develop an automated computer vision based system to facilitate forensic odonatological applications. These systems comprised of a variety of image processing techniques. The basic algorithms and methods used in dental $\mathrm{x}$-ray processing are image enhancement, image segmentation, edge detection with feature extraction and neural networks based classification. Teeth segmentation from dental $\mathrm{x}$-rays is an essential step for automating diagnosis as well as forensic procedures like postmortem identification [4].

Inclusion Criteria: The scope of this review covers general dental image processing methods, as well as a brief description of their clinical and forensic applications. The inclusion criteria for this review are recent original papers its applications to forensic odontology, accuracy. These are described in the Table 1. A summary of included image processing methods in the systemic review, used on dental radiographs and their purpose in forensic odontology is shown in Table 2.

Table 1: Inclusion criteria of the literature for this review.

\begin{tabular}{|c|}
\hline Inclusion criteria \\
\hline Original papers with full text availability online \\
Image processing methodologies applied to forensic odontology \\
Optimality of the algorithms proposed \\
Relevant recent studies between 2007 to 2017 \\
\hline
\end{tabular}


Table 2: Summary of included image processing methods used on dental radiographs and their purpose.

\begin{tabular}{|c|c|c|c|c|}
\hline Authors and year & Title & $\begin{array}{c}\text { Image processing } \\
\text { methods used }\end{array}$ & $\begin{array}{c}\text { Purpose of the study/ } \\
\text { Application }\end{array}$ & $\begin{array}{c}\text { Accuracy claimed by the } \\
\text { authors }\end{array}$ \\
\hline $\begin{array}{c}\text { PM Mahasantipiya, et al. } \\
{[10] 2011}\end{array}$ & $\begin{array}{c}\text { Bite Mark Identification } \\
\text { Using Neural Networks: A } \\
\text { Preliminary Study }\end{array}$ & $\begin{array}{c}\text { Contrast limit adaptive } \\
\text { histogram followed with } \\
\text { median filter }\end{array}$ & $\begin{array}{c}\text { Forensic bite mark } \\
\text { identification }\end{array}$ & 82\% \\
\hline PH Lira et al. [11] 2013, & $\begin{array}{c}\text { Using the mathematical } \\
\text { morphology and shape } \\
\text { matching for automatic data } \\
\text { extraction in dental X-ray } \\
\text { images. }\end{array}$ & $\begin{array}{c}\text { Mathematical morphology } \\
\text { of the teeth }\end{array}$ & $\begin{array}{c}\text { Automated forensic dental } \\
\text { Identification }\end{array}$ & Not reported \\
\hline R Cameriere, et al. [12] & $\begin{array}{c}\text { Automatic age estimation } \\
\text { in adults by analysis of } \\
\text { canine pulp/tooth ratio: } \\
\text { Preliminary results }\end{array}$ & $\begin{array}{c}\text { From the boundary of the } \\
\text { tooth and of the boundary } \\
\text { of the pulp, the area of } \\
\text { the tooth and the pulp is } \\
\text { calculated in terms of the } \\
\text { number of pixels inside the } \\
\text { corresponding zone. }\end{array}$ & $\begin{array}{c}\text { To assess dental age from } \\
\text { peri-apical x-rays }\end{array}$ & Not reported \\
\hline Jaffino G et al. [13]. 2017 & $\begin{array}{c}\text { A new mathematical } \\
\text { modelling based shape } \\
\text { extraction technique for } \\
\text { Forensic Odontology. }\end{array}$ & $\begin{array}{c}\text { Isoperimetric graph } \\
\text { partitioning method for } \\
\text { shape extraction }\end{array}$ & $\begin{array}{c}\text { Automated forensic dental } \\
\text { Identification }\end{array}$ & 97 $\%$ \\
\hline
\end{tabular}

Exclusion Criteria: Studies which had similar methods that were often used were excluded to prevent duplication. Only publications in English were reviewed. A list of data that was extracted from the reviewed full texts is shown in Table 3.

Table 3: The list of data that was extracted from the reviewed full texts.

\begin{tabular}{|c|}
\hline Data extracted from full text items \\
\hline Title and year \\
\hline Authors \\
\hline Purpose of the study/Application \\
\hline Image segmentation method \\
\hline Matching method \\
\hline Performance rate \\
\hline
\end{tabular}

Study Identification and Selection: The information used in this review were derived from well-known databases including the Directory of Open Access Journals (DOAJ), Medline/PubMed (NLM), ProQuest, Web of Science (Thomson), Science Direct Journals (Elsevier), Wiley (Cross Ref), Wiley Online library and Google Scholar, to assimilate information for this review. Review articles, reports and original papers published in peer journals, books, conference proceedings were all considered. Emphasis was placed on more recent literature from 2010 to-date. The emphasized search keywords used were dental image processing, image segmentation on dental radiographs for human identification from dental $\mathrm{x}$-rays and dental age estimation methods, image processing on human bite mark analysis and artificial neural networks on dental radiographs. The Preferred Reporting Items for Systematic reviews and Meta-analyses (PRISMA) guidelines [9-15] were used in this review.

Data Extraction and Management: The summary of the collected information was organized in a spread-sheet under the following categories: Author, year, title, enhancement technique, segmentation technique, feature extraction method, matching method, performance/accuracy and its applications. Figure 1 depicts the flowchart of the study selection in this review.

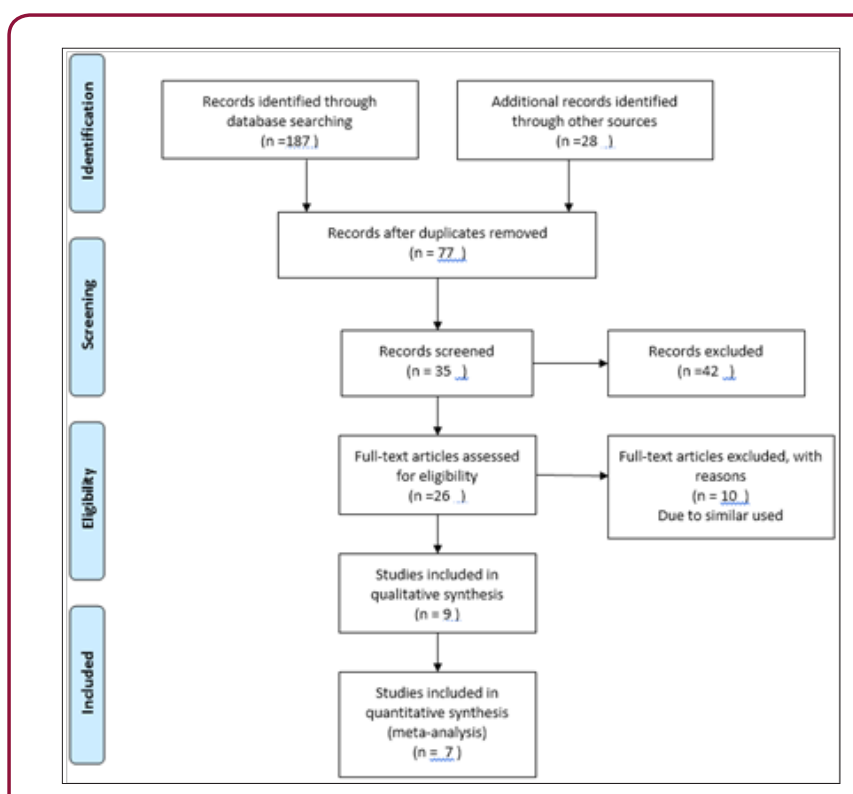

Figure 1: Flowchart of the study selection in this review.

\section{Image Processing Methods Used On Dental X-Ray Images}

Digital images are the most crucial medium in the field of computer vision. A digital radiograph provides the ability to apply special image processing techniques that enhance the overall display quality of the image and extract only the regions of interests. With image segmentation on digital dental radiographs, the exact information of the region of interest can be identified [10]. This information is an important tool in clinical, forensic and therapeutic applications in the field of dentistry. 


\section{Image Segmentation and Feature Extraction Techniques on Dental Radiographs}

A review on dental biometric systems and technology with further applications in forensic science was undertaken. The authors PM Mahasantipiya, et al. [10] described a preliminary study of bite mark identification using neural networks methodology. In this technique, each bite mark sample is transformed from bit depth of 32 to 8-bit gray scale, followed with stages like contrast limit adaptive histogram followed with median filter to enhance the tooth borders and several features like CC line(Distance from the centric point of the left canine to the right canine), I-LC angle(Angles between a straight line from centric point of each anterior tooth to centric point of the left canine and the CC line), I-RC angle(Angles between a straight line from centric point of each anterior tooth to centric point of the right canine and the CC line), curvature (The curvature of the parabola shape which is best fit the set of centric points of the anterior teeth.), iratio (Ratio of distances which is the shortest I-CC/ICC of each incisor teeth), HTT-D(Distance between two tooth centric points projected on the CC line) is computed. Gradient descending algorithm for adjusting weights and biased was employed in the training process with the help of LevenbergMarquardt algorithm to provide the numerical solution [10]. This ANN based work in this study was not able to differentiate the different bite marks correctly. The angles measured in this study used the tooth centric point that may not represent person's identity well which caused the poor identification.

PH Lira et al. [11] in 2013 used top-hat and bottom-hat morphological operations for image enhancement. Then, the Otsu's Thresholding was used to get raw teeth segmentation. This result was processed by a labeling technique for identification of the target teeth. In this stage, the binary image was eroded to get a set of seeds which are the input for a distance transform operator. From the obtained distance field a mask was generated by using the watershed operator. This mask was used to separate objects in the Otsu's result. In the final step of the segmentation, a morphological close operation was applied over the small regions remaining. The boundary of the obtained regions were extracted and aligned with a reference shape in order to perform the feature extraction [11]. The Figure 1 shows the experimental results of the method discussed in [11]. The Figure 2 describes their segmentation pipeline (Figures 2 \& 3 ).

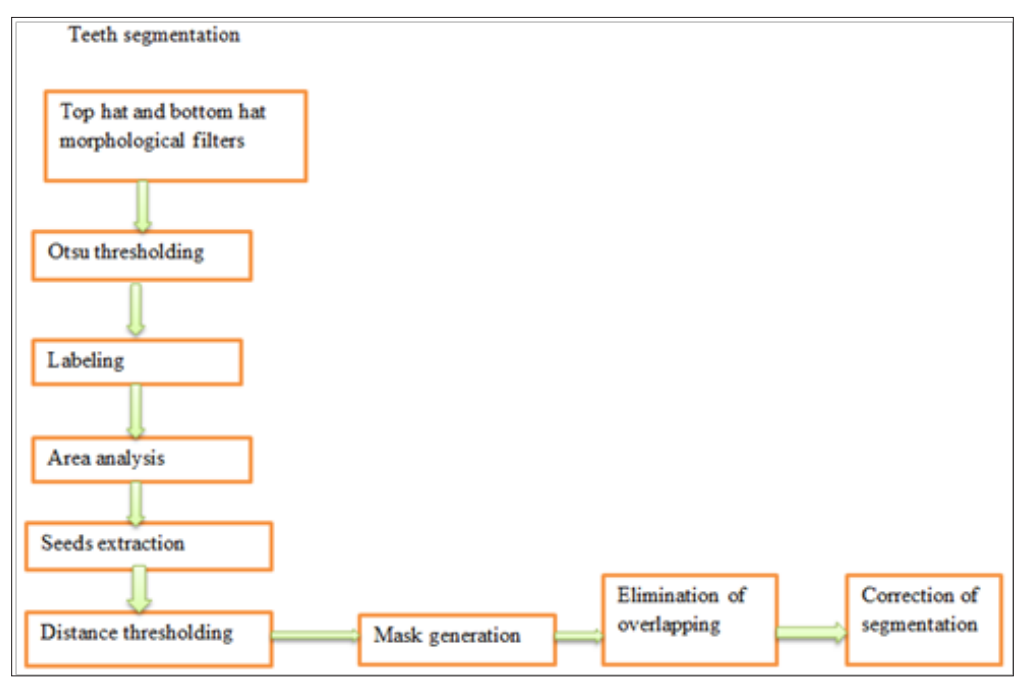

Figure 2: Describes the results of their proposed method PH Lira et al [11].

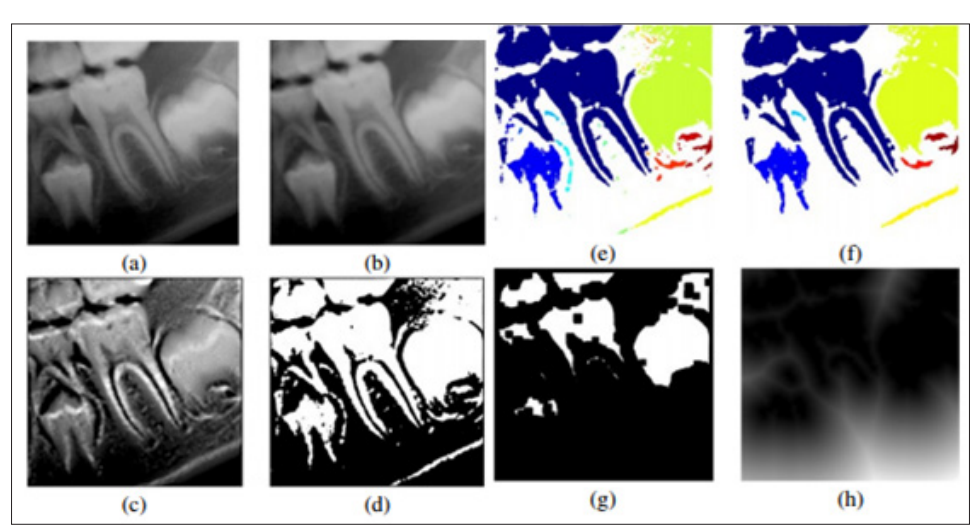

Figure 3: Results of the proposed Method of [11]. (a) Original image; (b) Gaussian Filter's result; (c) Combining top-hat and bottom-hat transforms according to expression (3); (d) Otsu's image (T=102); (e) Labelling's image; (f) The larger ones are selected in labeling result; (g) Seeds of teeth and (h) Distances transform field. 


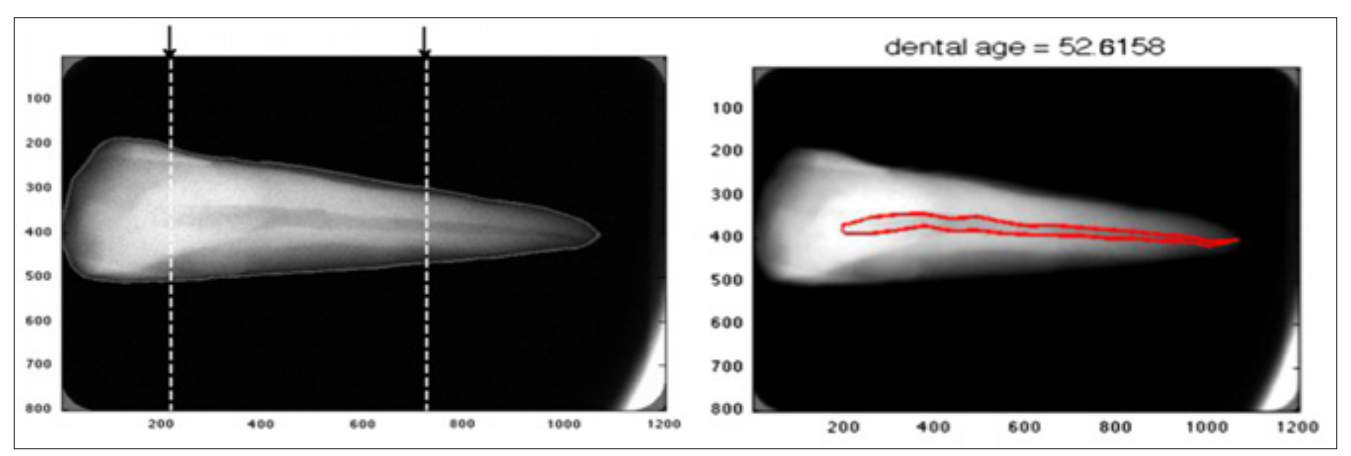

Figure 4: (a) Segmentation of teeth (b) Segmentation of the pulp area.

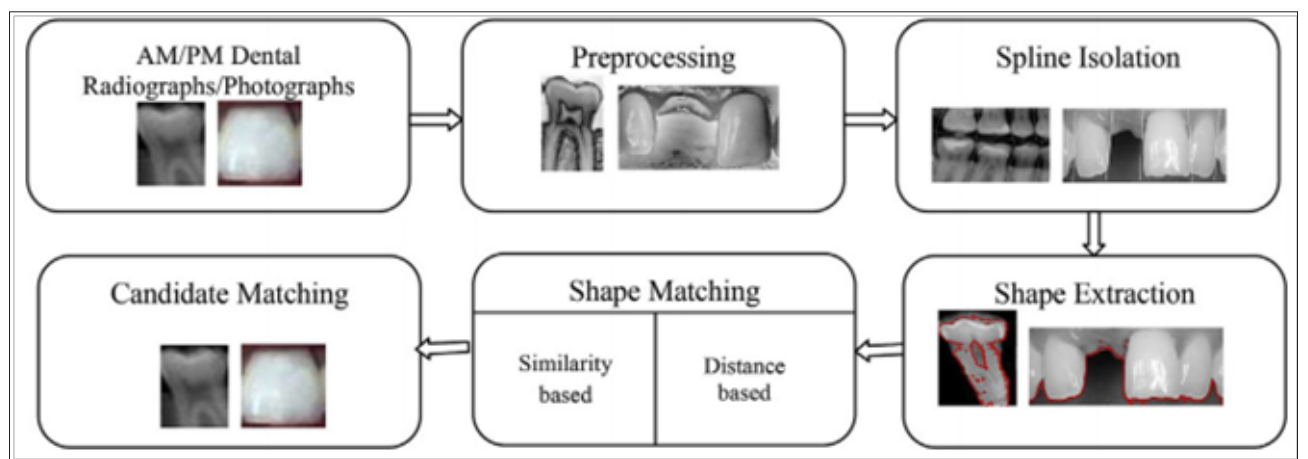

Figure 5: Proposed methodology in [13].

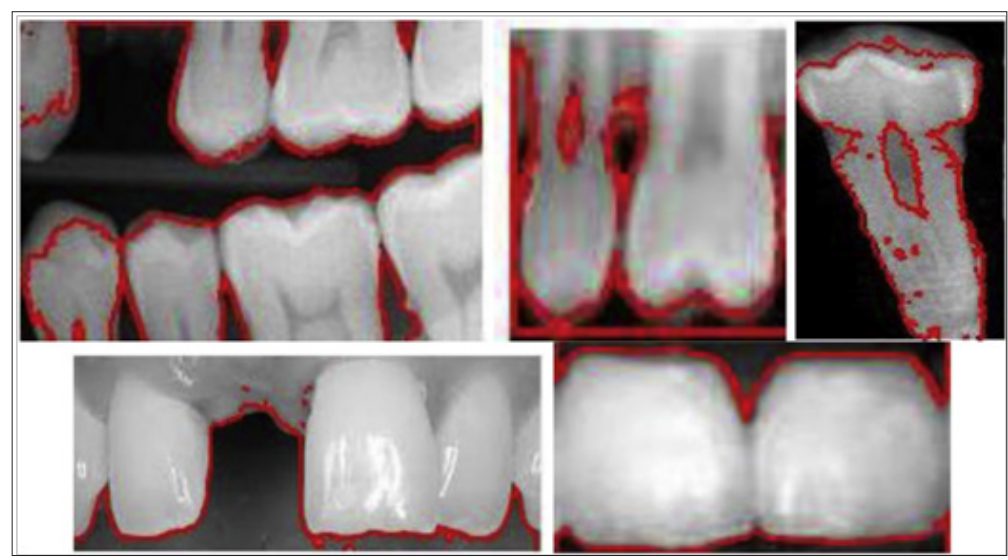

Figure 6: The isoperimetric output for radiographic and photographic images [13].

From the knowledge of the boundary of the tooth and of the boundary of the pulp the authors calculated the area of the tooth and of the pulp in terms of the number of pixels inside the corresponding zone. Jaffino G et al. [13] proposed an automatic algorithm for person identification from dental radiographs and photographs. In [13], texture based shape extraction algorithm is described for the analysis. For the shape matching, distance measure and similarity based matching was also added. Each tooth in the radiograph was classified using k-NN classifier with Hu's moment invariants as feature. Then each individual tooth was separated with pulp, enamel and dentine is applied to GLCM (gray-level co-occurrence matrix) texture features. The authors suggested isoperimetric graph partition algorithm to segment a region of an image with high volume and that shares a small perimeter with its surroundings gives the good segmentation, for shape extraction from dental images for forensic identification. Their results find that a better matching is observed by distance metric rather than similarity measures. Figure 5 shows the pipeline of their proposed methodology (Figures 5 \& 6). In [13] the authors claim

that the accuracy of $97 \%$ and $93 \%$ are obtained for radiographs and photographs respectively

\section{Discussion}

In teeth segmentation algorithms, optimality and percentage of failure indicate the performance of the segmentation algorithms. Measuring sub-optimality evaluated the performance of the algorithms in between the two extremes. In practical cases, it is difficult to achieve optimal performance with $100 \%$ of the images. As such, when comparing segmentation algorithms, one should 
favor those whose failure rates are the lowest and their optimality and low-order measures of sub-optimality predominate the testing results [14]. The failure rate is especially important when assessing teeth segmentation algorithms, since those images in which no teeth can be properly segmented cannot be used in the identification process. A summary of the main techniques explored in the literature with regards to dental image processing is given in Table 3. In the work of PH Lira et al. [10], the problem of overlap and the need to overcome is highlighted .From the review the work of Jaffino G, et al. [13] seems promising approach for teeth segmentation and matching by mathematical modelling based shape extraction as it claims 97\% accuracy in performance. The approach of R Cameriere, et al. [12], identifies there is a strong requirement of modification of segmentation algorithm for age estimation purpose to more precisely identify the regions of interests (Table 4).

Table 4: A comparison between the teeth segmentation algorithms.

\begin{tabular}{|c|c|}
\hline Authors & \multicolumn{1}{c}{ Principles } \\
\hline PH Lira et al. [11] 2013, & $\begin{array}{r}\text { Discrete shape model and shape analysis. Principal } \\
\text { analysis (PCA). }\end{array}$ \\
\hline $\begin{array}{c}\text { PM Mahasantipiya, et al. [10] } \\
\text { R Cameriere, et al. [12 ] 2015 }\end{array}$ & $\begin{array}{r}\text { From the boundary of the tooth and of the bound } \\
\text { pulp, the area of the tooth and the pulp is calculate } \\
\text { the number of pixels inside the corresponding }\end{array}$ \\
\hline Jaffino G, et al. [13 ] 2017 & Thresholding technique and shape analys \\
\hline
\end{tabular}

\section{Conclusion And Recommendations}

This systematic review summarizes and compares the results of some of the most commonly used methods for dental image segmentation in forensic application, mainly for human identification. A comprehensive overview of research works in

more than a decade in forensic odonatological image processing is presented here. From the review of above papers, the main challenge in developing an automated dental recognition system is to deal with poor quality of images, imaging angle, teeth overlap, teeth shape change (due to aging, accidents, etc.), occluded teeth and other issues. It is evident that despite a vast research effort, image segmentation on radiographic images continues to be extremely challenging. There is a need to identify teeth segmentation algorithms with better performance for further forensic and clinical applications. For feature extraction and segmentation, most research scholars make use of Thresholding and morphological operations [25-31].

Complex dental x-ray segmentation incorporated with artificial intelligence (AI) techniques such as feed-forward (associative) artificial neural networks (ANNs) can produce less noisy images and more accurate segmentation results [32]. Incorporating other AI tools such as fuzzy c-means clustering has also not been much explored for the better understanding and diagnosis of dental images. Researchers up till now have been found concentrating on image enhancement or segmentation to extract features for forensic sciences in human identification. No deep research has been published for automating the dental atlas in human age prediction for odonatological purposes. ANN based pattern recognition is an area which needed to worked upon in automating dental atlas for age estimation. Preprocessing and

pattern recognition of bite mark analysis using image processing and ANN needs to be well explored. Automated or semi-automated diagnosis of the aforesaid objectives would be useful for further identification of humans in a wide range applications including in the identification of asylum seekers and refugees [15-33].

\section{Acknowledgment}

The authors wish to acknowledge Riyadh elm University, Riyadh,Saudi Arabia for providing support for this research work.

\section{References}

1. Avon SL (2004) Forensic odontology: the roles and responsibilities of the dentist. J Can Dent Assoc 70(7): 453-458.

2. RC Gonzales, RE Woods (2002) Image segmentation, Digital Image Processing, (2 ${ }^{\text {nd }}$ Edition), Prentice Hall, Upper Saddle River, New Jersey, USA.

3. Omaima Nomir, Mohamed Abdel-Mottaleb (2005) A system for human identification from X-ray dental radiographs. Pattern Recognition 38(8): 1295-1305.

4. EH Said, DEM Nassar, G Fahmy, HH Ammar (2006) Teeth segmentation in digitized dental X-ray films using mathematical morphology. IEEE Transactions on information forensic and security 1(2): 178-189.

5. Flora G, Tuceryan M, Blitzer H (2009) Forensic bite mark identification using image processing methods. ACM symposium on Applied computing pp. 903-907.

6. PHM Lira, GA Giraldi, LAP Neves (2009) Panoramic Dental X-Ray Image Segmentation and Feature Extraction. V Workshop of Computing Vision, Sao Paulo, Brazil.

7. LAP Neves, G Giraldi, AG Costa, EC Kuchler, DEM Oliveira (2009) Automatic Data Extraction in Odontological X-Ray Imaging. VISAPP International Conference on Computer Vision Theory and Applications pp. 141-144.

8. PHM Lira, GA Giraldi, LAP Neves (2009) an Automatic Morphometrics Data Extraction Method in Dental X-Ray Image. I International Conference on Biodental Enginnering, Porto, Portugal.

9. Tuan TM, Ngan TT (2016) A novel semi-supervised fuzzy clustering method based on interactive fuzzy satisficing for dental x-ray image segmentation. Applied Intelligence 45(2): 402-428.

10. Mahasantipiya PM, Yeesarapat U, Suriyadet T, Sricharoen J, Dumrongwanich A, et al. (2011) Bite mark identification using neural networks: A preliminary study. In Proceedings of the International Multi Conference of Engineers and Computer Scientists.

11. Lira PH, Giraldi GA, Neves LA (2013) Using the mathematical morphology and shape matching for automatic data extraction in dental X-ray images. InIX Workshop de Visao Computacional.

12. R Cameriere, E Cunha, E Sassaroli, E Nuzzolese, L Ferrante (2009) Age estimation by pulp/tooth area ratio in canines: study of a Portuguese sample to test Cameriere's method. Forensic Sci Int 193(1-3): 128e1$128 \mathrm{e} 6$. 
13. Jaffino G, Banumathi A, Gurunathan U, Vijayakumari B (2017) A new mathematical modelling based shape extraction technique for Forensic Odontology. Journal of Forensic and Legal Medicine 47: 39-45.

14. P Marquez-Neila, L Baumela, L Alvarez (2014) A Morphological Approach to Curvature-Based Evolution of Curves and Surfaces. IEEE Transactions on Pattern Analysis and Machine Intelligence 36(1): 2-17.

15. M Abdel-Mottaleb, O Nomir, D Nassar, G Fahmy, H Ammar (2003) Challenges of Developing an Automated Dental Identification System. IEEE 46th Midwest Symposium on Circuits and Systems. pp. 411-414.

16. F Shamsafar (2013) A New Feature Extraction Method From Dental X-ray Images for Human Identification. 8th Iranian Conference on Machine Vision and Image Processing (MVIP) pp. 397-402.

17. HHA Diaa Eldin, M Nassar (2003) A Prototype Automated Dental Identification System (ADIS).

18. G Fahmy, D Nassar, E Haj-Said, H Chen, O Nomir, et al. (2004) Towards an Automated Dental Identification System (ADIS). In: D Zhang, AK Jain (Eds.). Biometric Authentication, ser. Lecture Notes in Computer Science Springer Berlin Heidelberg, pp. 789-796.

19. M Abdel-Mottaleb, O Nomir, D Nassar, G Fahmy, H Ammar (2003) Challenges of Developing an Automated Dental Identification System. IEEE $46^{\text {th }}$ Midwest Symposium on Circuits and Systems pp. 411-414.

20. J Zhou, M Abdel-Mottaleb (2005) A Content-based System for Human Identification Based on Bitewing Dental X-ray Images. Pattern Recognition 38(11): 2132-2142.

21. E Said, D Nassar, G Fahmy, H Ammar (2006) Teeth Segmentation in Digitized Dental X-ray Films Using Mathematical Morphology. IEEE Transactions on Information Forensics and Security 1(2): 178-189.

22. M Mahoor, M Abdel-Mottaleb (2004) Automatic Classification of Teeth in Bitewing Dental Images. 5: 3475-3478.

23. E Said, GF Fahmy, D Nassar, H Ammar (2004) Dental X-ray Image Segmentation. 5404: 409-417.

24. J Zhou, M Abdel-Mottaleb (2004) Automatic Human Identification Based on Dental X-ray Images. 5404: 373-380.

25. Cameriere R, De Luca S, Egidi N, Bacaloni M, Maponi P, et al. (2015) Automatic age estimation in adults by analysis of canine pulp/tooth ratio: preliminary results. Journal of Forensic Radiology and Imaging 3(1): 61-66.

26. AK Jain, H Chen (2004) Matching of Dental X-ray Images for Human Identification. Pattern Recognition 37(7): 1519-1532.

27. Moher D, Liberati A, Tetzlaff J, Altman DG (2009) Preferred Reporting Items for Systematic Reviews and Meta-Analyses: The PRISMA Statement. BMJ 339: b2535.

28. Acharya T, Ray AK (2010) Image processing: principles and applications. John Wiley \& Sons.

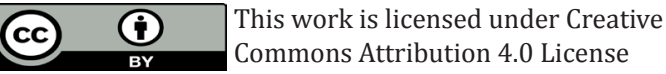

Submission Link: https://biomedres.us/submit-manuscript.php
29. Parker JR (2010) Algorithms for image processing and computer vision. John Wiley \& Sons.

30.Van der Stelt PF (2008) Better imaging: the advantages of digital radiography. The Journal of the American Dental Association 139: S7-13.

31. Yao X (1999) Evolving artificial neural networks. Proceedings of the IEEE 87(9): 1423-1447.

32. Acharya T, Ray AK (2005) Image processing: principles and applications. John Wiley \& Sons.

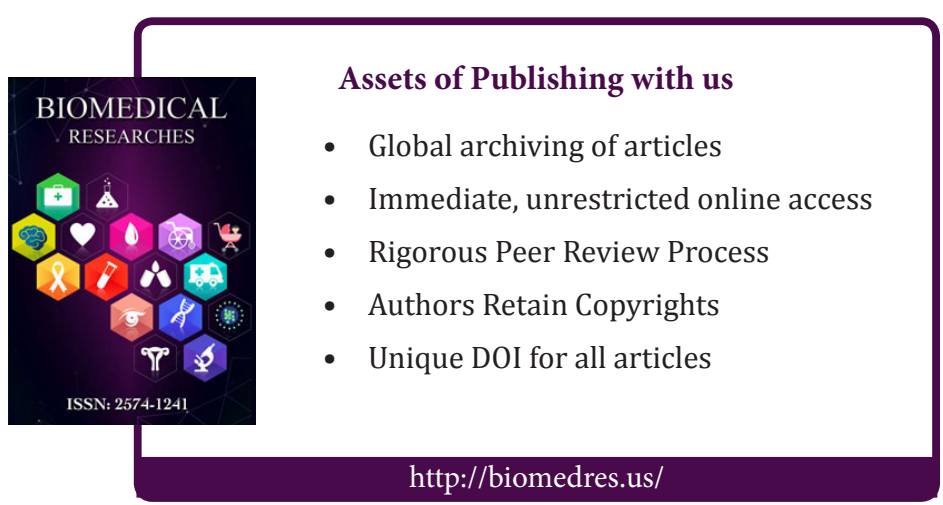

